PROCEEDINGS OF THE

AMERICAN MATHEMATICAL SOCIETY

Volume 126, Number 6, June 1998, Pages 1739-1746

S $0002-9939(98) 04247-6$

\title{
ON WEIGHTED WEAK TYPE INEQUALITIES FOR MODIFIED HARDY OPERATORS
}

\author{
F. J. MARTÍN-REYES AND P. ORTEGA
}

(Communicated by J. Marshall Ash)

\begin{abstract}
We characterize the pairs of weights $(w, v)$ for which the modified Hardy operator $T f(x)=g(x) \int_{0}^{x} f$ applies $L^{p}(v)$ into weak- $L^{q}(w)$ where $g$ is a monotone function and $1 \leq q<p<\infty$.
\end{abstract}

\section{INTRODUCTION}

The purpose of this paper is to study weighted weak type inequalities for the modified Hardy operator $T$ acting on measurable functions $f:(0, \infty) \rightarrow \mathbb{R}$ and defined by

$$
T f(x)=g(x) \int_{0}^{x} f(y) d y,
$$

where $g$ is a nonnegative measurable function on $(0, \infty)$. Examples of these operators are the Hardy operator $\frac{1}{x} \int_{0}^{x} f(y) d y$ and the operators $x^{\alpha} \int_{0}^{x} f(y) d y$ which have been extensively studied in the last twenty years.

Muckenhoupt $[\mathrm{Mu}]$ and Bradley $[\mathrm{B}]$ studied the weighted strong type inequality $(p, q)$ with $1 \leq p \leq q$ in the case $g(x)=1$. If $w$ and $v$ are nonnegative measurable functions then the result in $[\mathrm{B}]$ implies directly that the weighted strong type inequality

$$
\left(\int_{0}^{\infty}|T f|^{q} w\right)^{1 / q} \leq C\left(\int_{0}^{\infty}|f|^{p} v\right)^{1 / p} \quad \text { for all } f \in L^{p}(v)
$$

holds for $1 \leq p \leq q$ if and only if

$$
\sup _{a>0}\left(\int_{a}^{\infty} g^{q} w\right)^{1 / q}\left(\int_{0}^{a} v^{1-p^{\prime}}\right)^{1 / p^{\prime}}<\infty .
$$

However, the characterizations of the weighted weak type inequalities

$$
\lambda\left(\int_{\{|T f|>\lambda\}} w\right)^{1 / q} \leq C\left(\int_{0}^{\infty}|f|^{p} v\right)^{1 / p}
$$

Received by the editors September 8, 1995 and, in revised form, December 1, 1996.

1991 Mathematics Subject Classification. Primary 26D15.

Key words and phrases. Hardy operators, weights, inequalities.

This research has been partially supported by D.G.I.C.Y.T. grant (PB94-1496) and Junta de Andalucía. 
for $1 \leq p \leq q$ and general $g$ cannot be deduced directly from the corresponding ones for $g(x)=1$. These characterizations can be found in [F], [AM], [S], [MOS]. Weighted weak type inequalities for $p<q$ and an operator of similar generality have been studied in [MT].

We are interested in the case $1 \leq q<p<\infty$. As before, the weighted strong type inequality (1) for general $g$ can be obtained from Maz'ja's result [Ma] which characterizes the pairs of weights if $g=1$. In this way it is proved that if $1 \leq q<$ $p<\infty$ then (1) holds if and only if

$$
\left(\int_{0}^{\infty}\left[\left(\int_{x}^{\infty} g^{q} w\right)^{1 / q}\left(\int_{0}^{x} v^{1-p^{\prime}}\right)^{1 / q^{\prime}}\right]^{r} v^{1-p^{\prime}}(x) d x\right)^{1 / r}<\infty,
$$

where $1 / r=1 / q-1 / p$ and $1 / p+1 / p^{\prime}=1=1 / q+1 / q^{\prime}$, which clearly means that the function

$$
x \mapsto\left(\int_{x}^{\infty} g^{q} w\right)^{1 / q}\left(\int_{0}^{x} v^{1-p^{\prime}}\right)^{1 / q^{\prime}}
$$

belongs to $L^{r}\left(v^{1-p^{\prime}}\right)$. By duality arguments or integrating by parts, one can see that the above condition is equivalent to the fact that the function

$$
\Lambda(x)=g^{q / r}(x)\left(\int_{x}^{\infty} g^{q} w\right)^{1 / p}\left(\int_{0}^{x} v^{1-p^{\prime}}\right)^{1 / p^{\prime}}
$$

belongs to $L^{r}(w)$. Therefore, the characterization of the weighted strong type inequality for $T$ can be stated as follows:

Theorem A ([Ma]). Let $1 \leq q<p<\infty$ and let $w, v, g, T$ and $\Lambda$ be as above. Then (1) holds if and only if $\Lambda \in L^{r}(w)$.

As far as we know, the problem of characterizing the pairs of weights for which inequality (2) holds in the case $1 \leq q<p<\infty$ is not solved even if $g(x)=1 / x$. Our results solve this problem for monotone $g$. If $g$ is a monotone function we are able to give an easy characterization which is related to the condition in Theorem A. This characterization is in the following theorem. Before stating the result, we need some notation: if $f$ is a measurable function on $(0, \infty)$ we write

$$
\|f\|_{r, \infty ; w}=\sup _{\lambda>0} \lambda\left(\int_{\{|f|>\lambda\}} w\right)^{1 / r}
$$

and we denote by $L_{r, \infty}(w)$ the set of all measurable functions such that $\|f\|_{r, \infty ; w}$ is finite.

Theorem 1. Let $1 \leq q<p<\infty$ and let $r, w, v, g$ and $T$ be as above. Assume also that $g$ is a monotone function on $(0, \infty)$. Then the following statements are equivalent:

(a) There exists $C>0$ such that

$$
\lambda\left(\int_{\{|T f|>\lambda\}} w\right)^{1 / q} \leq C\left(\int_{0}^{\infty}|f|^{p} v\right)^{1 / p}
$$

for all $\lambda>0$ and all $f \in L^{p}(v)$. 
(b) The function $\Phi:(0, \infty) \rightarrow \mathbb{R}$ defined by

$$
\Phi(x)=\sup _{0<a<x<b}\left[\inf _{y \in(a, b)} g(y)\left(\int_{a}^{b} w\right)^{1 / p}\left(\int_{0}^{a} v^{1-p^{\prime}}\right)^{1 / p^{\prime}}\right]
$$

belongs to $L_{r, \infty}(w)$.

(c) The function $\Psi:(0, \infty) \rightarrow \mathbb{R}$ defined by

$$
\Psi(x)=\sup _{b>x}\left[\inf _{y \in(x, b)} g(y)\left(\int_{x}^{b} w\right)^{1 / p}\left(\int_{0}^{x} v^{1-p^{\prime}}\right)^{1 / p^{\prime}}\right]
$$

belongs to $L_{r, \infty}(w)$.

Remarks. (1) Let $\Psi$ be the function in Theorem 1. If $g$ is decreasing then

$$
\Psi(x)=\sup _{b>x} g(b)\left(\int_{x}^{b} w\right)^{1 / p}\left(\int_{0}^{x} v^{1-p^{\prime}}\right)^{1 / p^{\prime}} ;
$$

if $g$ is increasing then

$$
\Psi(x)=g(x)\left(\int_{x}^{\infty} w\right)^{1 / p}\left(\int_{0}^{x} v^{1-p^{\prime}}\right)^{1 / p^{\prime}} \quad \text { a.e. }
$$

(2) Let $g$ be a monotone function. If $\Lambda$ and $\Psi$ are the functions in Theorems A and 1 respectively, then $\Psi \leq \Lambda$ and therefore $\Lambda \in L^{r}(w)$ implies directly condition (c) in Theorem 1, i.e., the condition characterizing the strong type inequality implies easily the condition characterizing the weak type inequality.

Our characterization allows to give non-trivial examples of pairs of weights for which the operator $T$ is of weak type but is not of strong type.

Example. Let $2<q<p<\infty$ with $p q-2 q-2 p+4 \neq 0, g(x)=1 / x, w(x)=x$, and $v(x)=x^{\frac{s}{1-p^{\prime}}}$ with $s=p\left(1-\frac{2}{r}\right)-3$. Consider the functions $\Lambda$ and $\Psi$ in Theorems A and 1. Then, up to constants, $\Lambda(x)=x^{\gamma}$, where $\gamma=\frac{2}{p}-1+(p-1)\left(1-\frac{2}{q}\right)$ and $\Psi(x)=x^{-\frac{2}{r}}$. It is clear that $\Lambda \notin L^{r}(w)$. On the other hand, it is easy to see that a potential function $x^{\alpha}$ belongs to $L_{r, \infty}(w)$ if and only if $\alpha=-\frac{2}{r}$. Therefore, $\Psi \in L_{r, \infty}(w)$ and $\Lambda \notin L_{r, \infty}(w)$. These facts imply that the operator $T f(x)=x^{-1} \int_{0}^{x} f$ is not of strong type $(p, q)$ but it is of weak type $(p, q)$ with respect to the measures given by the weights $w$ and $v$.

Moreover, the preceding example shows that the weighted weak type $(p, q)$ inequality for $T$ is not characterized by the fact that $\Lambda \in L_{r, \infty}(w)$, as could be expected after Theorem A.

If $g$ is increasing then the fact that $h(x)=\int_{0}^{x} f$ is also increasing allows us to obtain an easier condition with a simpler proof. This appears in the following theorem:

Theorem 2. Let $1 \leq q<p<\infty$ and let $r, w, v, g$ and $T$ be as above. Assume also that $g$ is an increasing function on $(0, \infty)$. Then the following statements are equivalent: 
(a) There exists $C>0$ such that

$$
\lambda\left(\int_{\{|T f|>\lambda\}} w\right)^{1 / q} \leq C\left(\int_{0}^{\infty}|f|^{p} v\right)^{1 / p}
$$

for all $\lambda>0$ and all $f \in L^{p}(w)$.

(b) The function $\tilde{\Psi}:(0, \infty) \rightarrow \mathbb{R}$ defined by

$$
\tilde{\Psi}(x)=g(x)\left(\int_{x}^{\infty} w\right)^{1 / q}\left(\int_{0}^{x} v^{1-p^{\prime}}\right)^{1 / p^{\prime}}
$$

belongs to $L^{\infty}$.

Observe that Theorem 1 solves the problem for the modified Hardy operators considered in $[\mathrm{AM}]$ and $[\mathrm{S}]$.

The proofs of the theorems appear in the following sections. In these proofs we use ideas of the paper [Q] where weighted weak type inequalities $(p, q)$ with $q<p$ have been studied. We also use some techniques that were introduced in $[\mathrm{M}]$ which have been already applied in [MOS].

It is worth noting that similar results can be obtained for the operator $g(x) \int_{x}^{\infty} f$.

Throughout the paper, the letter $C$ will mean a positive constant not necessarily the same at each occurrence.

\section{Proof of Theorem 1}

(a) $\Rightarrow(\mathrm{b})$. We may assume that $w$ and $v^{1-p^{\prime}}$ are integrable functions since the general case follows from this by a limit argument.

We have to prove that

$$
\sup _{\lambda>0} \lambda\left(\int_{\{x \in(0, \infty): \Phi(x)>\lambda\}} w\right)^{1 / r}<\infty .
$$

Let $\lambda>0$ and $S_{\lambda}=\{x \in(0, \infty): \Phi(x)>\lambda\}$. For every $z \in S_{\lambda}$ there exist $a_{z}$ and $b_{z}$ with $0<a_{z}<z<b_{z}$ such that

$$
\left(\int_{a_{z}}^{b_{z}} w\right)^{1 / p}\left(\int_{0}^{a_{z}} v^{1-p^{\prime}}\right)^{1 / p^{\prime}} \inf _{y \in\left(a_{z}, b_{z}\right)} g(y)>\lambda .
$$

Let $K$ be a compact set contained in $S_{\lambda}$. Then there exist $\left(a_{z_{1}}, b_{z_{1}}\right), \ldots,\left(a_{z_{k}}, b_{z_{k}}\right)$ which cover $K$. We may assume that $\sum_{j} \chi_{\left(a_{z_{j}}, b_{z_{j}}\right)} \leq 2 \chi_{\cup_{j}\left(a_{z_{j}}, b_{z_{j}}\right)}$.

Let $f$ be the function on $(0, \infty)$ defined by

$$
f(x)=\left(\sum_{j=1}^{k}\left(\frac{1}{\inf _{y \in\left(a_{z_{j}}, b_{z_{j}}\right)} g(y) \int_{0}^{a_{z_{j}}} v^{1-p^{\prime}}}\right)^{p} v^{-p^{\prime}}(x) \chi_{\left(0, a_{z_{j}}\right)}(x)\right)^{1 / p} .
$$

If $z \in\left(a_{z_{j}}, b_{z_{j}}\right)$, we have

$$
T f(z) \geq g(z) \int_{0}^{a_{z_{j}}} \frac{1}{\inf _{y \in\left(a_{z_{j}}, b_{z_{j}}\right)} g(y) \int_{0}^{a_{z_{j}}} v^{1-p^{\prime}}} v^{1-p^{\prime}}(x) d x \geq 1 .
$$


Therefore, the set $\cup_{j=1}^{k}\left(a_{z_{j}}, b_{z_{j}}\right)$ is contained in the set $\{x \in(0, \infty): \operatorname{Tf}(x) \geq 1\}$. Then the weak type inequality in (a) and (3) gives

$$
\begin{aligned}
\int_{\cup_{j=1}^{k}\left(a_{z_{j}}, b_{z_{j}}\right)} w & \leq C\left(\sum_{j=1}^{k} \frac{1}{\left(\inf _{y \in\left(a_{z_{j}}, b_{z_{j}}\right)} g(y)\right)^{p}\left(\int_{0}^{a_{z_{j}}} v^{1-p^{\prime}}\right)^{p-1}}\right)^{q / p} \\
& \leq \frac{C}{\lambda^{q}}\left(\sum_{j=1}^{k} \int_{a_{z_{j}}}^{b_{z_{j}}} w\right)^{q / p} \leq \frac{C}{\lambda^{q}}\left(\int_{\cup_{j=1}^{k}\left(a_{z_{j}}, b_{z_{j}}\right)} w\right)^{q / p},
\end{aligned}
$$

i.e.,

$$
\lambda\left(\int_{\cup_{j=1}^{k}\left(a_{z_{j}}, b_{z_{j}}\right)} w\right)^{1 / r} \leq C .
$$

Since $K$ is contained in $\cup_{j=1}^{k}\left(a_{z_{j}}, b_{z_{j}}\right)$, we get $\lambda\left(\int_{K} w\right)^{1 / r} \leq C$. This inequality holds for every compact $K$ contained in $S_{\lambda}$. Therefore, $\lambda\left(\int_{S_{\lambda}} w\right)^{1 / r} \leq C$, as we wished to prove.

(b) $\Rightarrow($ c). It suffices to observe that $\Psi(x) \leq \Phi(x)$ a.e. In fact, if $g$ is continuous at $x$ and $b>x$ we have

$$
\begin{aligned}
\inf _{y \in(x, b)} g(y)\left(\int_{x}^{b} w\right)^{1 / p} & \left(\int_{0}^{x} v^{1-p^{\prime}}\right)^{1 / p^{\prime}} \\
& =\lim _{a \rightarrow x^{-}} \inf _{y \in(a, b)} g(y)\left(\int_{a}^{b} w\right)^{1 / p}\left(\int_{0}^{a} v^{1-p^{\prime}}\right)^{1 / p^{\prime}} \leq \Phi(x) .
\end{aligned}
$$

(c) $\Rightarrow$ (a). Let us suppose that $\Psi \in L_{r, \infty}(w)$. It suffices to prove (a) for nonnegative functions $f \in L^{1}$ such that $\int_{0}^{\infty} f^{p} v=1$. Let $\lambda>0$ and $O_{\lambda}=\{x \in(0, \infty)$ : $T f(x)>\lambda\}$. Then

$$
\int_{O_{\lambda}} w=\int_{O_{\lambda} \cap\left\{x \in(0, \infty): \Psi(x)>\lambda^{q / r}\right\}} w+\int_{O_{\lambda} \cap\left\{x \in(0, \infty): \Psi(x) \leq \lambda^{q / r}\right\}} w .
$$

By the definition of $\|\Psi\|_{r, \infty ; w}$, the first term on the right-hand side can be dominated as follows:

$$
\int_{O_{\lambda} \cap\left\{x \in(0, \infty): \Psi(x)>\lambda^{q / r}\right\}} w \leq \frac{\|\Psi\|_{r, \infty ; w}^{r}}{\lambda^{q}} .
$$

To estimate the second term, let us consider the decreasing sequence $\left\{x_{k}\right\}$ defined by $x_{0}=\infty$ and $\int_{x_{k+1}}^{x_{k}} f=\int_{0}^{x_{k+1}} f$. Then we have that

$$
\int_{0}^{x_{k}} f=4 \int_{x_{k+2}}^{x_{k+1}} f
$$

for all $k$. For each $k$, let $E_{k}=\left(x_{k+1}, x_{k}\right) \cap O_{\lambda} \cap\left\{x: \Psi(x) \leq \lambda^{q / r}\right\}, \alpha_{k}=\inf E_{k}$ and $\beta_{k}=\sup E_{k}$.

Observe that if $x \in E_{k}$ then

$$
\lambda<g(x) \int_{0}^{x} f \leq g(x) \int_{0}^{x_{k}} f=4 g(x) \int_{x_{k+2}}^{x_{k+1}} f .
$$


Therefore

$$
\lambda \leq 4 \inf _{z \in\left(\alpha_{k}, \beta_{k}\right)} g(z) \int_{x_{k+2}}^{x_{k+1}} f .
$$

(We are using that the monotonicity of $g$ implies that $\inf _{z \in E} g(z) \leq \inf _{z \in(\alpha, \beta)} g(z)$ where $\alpha=\inf E$ and $\beta=\sup E$.) Now, for each $k$, let $\left\{z_{n}^{k}\right\}$ be a decreasing sequence such that $\lim z_{n}^{k}=\alpha_{k}$ and $z_{n}^{k} \in E_{k}$ for all $n$. Then, by Hölder's inequality and (4), we obtain

$$
\begin{aligned}
\lambda & \leq 4 \inf _{z \in\left(\alpha_{k}, \beta_{k}\right)} g(z) \int_{x_{k+2}}^{x_{k+1}} f \leq 4 \inf _{z \in\left(z_{n}^{k}, \beta_{k}\right)} g(z) \int_{x_{k+2}}^{x_{k+1}} f \\
& \leq 4 \inf _{z \in\left(z_{n}^{k}, \beta_{k}\right)} g(z)\left(\int_{x_{k+2}}^{x_{k+1}} f^{p} v\right)^{1 / p}\left(\int_{x_{k+2}}^{x_{k+1}} v^{1-p^{\prime}}\right)^{1 / p^{\prime}} \\
& \leq 4 \inf _{z \in\left(z_{n}^{k}, \beta_{k}\right)} g(z)\left(\int_{x_{k+2}}^{x_{k+1}} f^{p} v\right)^{1 / p}\left(\int_{0}^{z_{n}^{k}} v^{1-p^{\prime}}\right)^{1 / p^{\prime}} .
\end{aligned}
$$

Multiplying by $\left(\int_{z_{n}^{k}}^{\beta_{k}} w\right)^{1 / p}$ we obtain

$$
\lambda\left(\int_{z_{n}^{k}}^{\beta_{k}} w\right)^{1 / p} \leq 4 \Psi\left(z_{n}^{k}\right)\left(\int_{x_{k+2}}^{x_{k+1}} f^{p} v\right)^{1 / p},
$$

and since $z_{n}^{k} \in E_{k}$ we get

$$
\lambda\left(\int_{z_{n}^{k}}^{\beta_{k}} w\right)^{1 / p} \leq 4 \lambda^{q / r}\left(\int_{x_{k+2}}^{x_{k+1}} f^{p} v\right)^{1 / p} .
$$

Keeping in mind that $\lim z_{n}^{k}=\alpha_{k}=\inf E_{k}$ and $\beta_{k}=\sup E_{k}$, the last inequality implies

$$
\int_{E_{k}} w \leq \frac{4^{p}}{\lambda^{q}} \int_{x_{k+2}}^{x_{k+1}} f^{p} v
$$

Summing up in $k$,

$$
\int_{O_{\lambda} \cap\left\{x: \Psi(x) \leq \lambda^{q / r}\right\}} w=\sum_{k} \int_{E_{k}} w \leq \frac{4^{p}}{\lambda^{q}} \sum_{k} \int_{x_{k+2}}^{x_{k+1}} f^{p} v \leq \frac{4^{p}}{\lambda^{q}} .
$$

Finally, putting together all the inequalities, we obtain

$$
\int_{O_{\lambda}} w \leq \frac{1}{\lambda^{q}}\left(4^{p}+\|\Psi\|_{L_{r, \infty}(w)}^{r}\right)
$$

as we wished to prove.

\section{Proof of Theorem 2}

In order to prove that (a) implies (b) we test the weak type inequality for the functions $f=v^{1-p^{\prime}} \chi_{(0, x)}$. Assume first that the functions $v^{1-p^{\prime}} \chi_{(0, x)}$ belong to $L^{1}$. If $z>x$ we have

$$
T f(z) \geq g(z) \int_{0}^{x} v^{1-p^{\prime}} \geq g(x) \int_{0}^{x} v^{1-p^{\prime}} .
$$


Then, by (a), we obtain

$$
g(x) \int_{0}^{x} v^{1-p^{\prime}}\left(\int_{x}^{\infty} w\right)^{1 / q} \leq C\left(\int_{0}^{\infty} f^{p} v\right)^{1 / p}=C\left(\int_{0}^{x} v^{1-p^{\prime}}\right)^{1 / p}
$$

which gives (b) under the assumption that the functions $v^{1-p^{\prime}} \chi_{(0, x)}$ belong to $L^{1}$. If this is not the case then an easy limit argument shows that (b) holds. Suppose now that (b) holds and let $f \in L^{p}(v)$. We may assume without loss of generality that $f$ is nonnegative and has compact support. Let $\lambda>0$ and $O_{\lambda}=\{x: T f(x)>\lambda\}$. Since $g$ is increasing, it turns out that $O_{\lambda}$ is an interval of the form $(a, \infty)$ or $[a, \infty)$ with $a>0$. Let us take a strictly decreasing sequence $\left\{z_{n}\right\}$ such that $\lim z_{n}=a$ and $\tilde{\Psi}\left(z_{n}\right) \leq\|\tilde{\Psi}\|_{L^{\infty}}$. Then, by Hölder's inequality,

$$
\lambda<g\left(z_{n}\right) \int_{0}^{z_{n}} f \leq g\left(z_{n}\right)\left(\int_{0}^{z_{n}} f^{p} v\right)^{1 / p}\left(\int_{0}^{z_{n}} v^{1-p^{\prime}}\right)^{1 / p^{\prime}},
$$

and therefore, by (b),

$$
\begin{aligned}
\lambda\left(\int_{z_{n}}^{\infty} w\right)^{1 / q} & \leq g\left(z_{n}\right)\left(\int_{z_{n}}^{\infty} w\right)^{1 / q}\left(\int_{0}^{z_{n}} v^{1-p^{\prime}}\right)^{1 / p^{\prime}}\left(\int_{0}^{z_{n}} f^{p} v\right)^{1 / p} \\
& \leq \tilde{\Psi}\left(z_{n}\right)\left(\int_{0}^{z_{n}} f^{p} v\right)^{1 / p} \leq\|\tilde{\Psi}\|_{L^{\infty}}\left(\int_{0}^{z_{n}} f^{p} v\right)^{1 / p} .
\end{aligned}
$$

Now, letting $n$ tend to $\infty$ we obtain

$$
\lambda\left(\int_{O_{\lambda}} w\right)^{1 / q} \leq\|\tilde{\Psi}\|_{L^{\infty}}\left(\int_{0}^{\infty} f^{p} v\right)^{1 / p},
$$

which gives (a).

It is worth noting that Theorem 2 can be proved via Theorem 1 by showing that the function $\Psi$ defined in Theorem 1 belongs to $L_{r, \infty}(w)$ if and only if $\tilde{\Psi}$ is in $L^{\infty}$.

\section{REFERENCES}

[AM] K. F. Andersen and B. Muckenhoupt, Weighted weak type Hardy inequalities with applications to Hilbert transforms and maximal functions, Studia Math. 72 (1982), 9-26. MR 83k:42018

[B] J. Bradley, Hardy inequalities with mixed norms, Canad. Math. Bull. 21 (1978), 405-408. MR 80a:26005

[F] E. V. Ferreyra, Weighted Lorentz norm inequalities for integral operators, Studia Math 96 (1990), 125-134. MR 91c:26025

[M] F. J. Martín-Reyes, New proofs of weighted inequalities for the one sided Hardy-Littlewood maximal functions, Proc. Amer. Math. Soc. 117 (1993), 691-698. MR 93d:42016

[MOS] F. J. Martín-Reyes, P. Ortega Salvador and M. D. Sarrión Gavilán, Boundedness of operators of Hardy type in $\Lambda^{p, q}$ spaces and weighted mixed inequalities for singular integral operators, Proc. Roy. Soc. Edinburgh Sect. A 127 (1997), 157-170. CMP 97:08

[MT] F. J. Martín-Reyes and A. de la Torre, Some weighted inequalities for general one-sided maximal operators, Studia Math. 122 (1997), 1-14. CMP 97:06

[Ma] V. G. Maz'ja, Sobolev Spaces, Springer-Verlag Berlin Heidelberg New York Tokyo, 1985. MR 87g:46056

[Mu B. Muckenhoupt, Hardy's inequality with weights, Studia Math. 44 (1972), 31-38. MR 47:418 
[Q] Lai Qinsheng, A note on the weighted norm inequality for the one-sided maximal operator, Proc. Amer. Math. Soc. 124 (1996), 527-537. MR 96d:42029

[S] E. T. Sawyer, Weighted Lebesgue and Lorentz norm inequalities for the Hardy operator, Trans. Amer. Math. Soc. 281 (1984), 329-337. MR 85f:26013

Análisis Matemático, Facultad de Ciencias, Universidad de Málaga, 29071 Málaga, SPAIN

E-mail address: martin@anamat.cie.uma.es

E-mail address: ortega@anamat.cie.uma.es 